\title{
Polarimetric Measurement of Jones Matrix of a Twisted Nematic Liquid Crystal Spatial Light Modulator
}

\author{
Tsogvoo Khos-Ochir ${ }^{1,3}$, Purevdorj Munkhbaatar ${ }^{2,3}$, Byung Kwan Yang ${ }^{4}$, Hyun Woo Kim ${ }^{2,3}$, \\ Jin Seung Kim ${ }^{1,2,3}$, and Kim Myung-Whun ${ }^{1,2,3} *$ \\ ${ }^{1}$ Department of Nanoscience and Technology, Chonbuk National University, Jeon-ju 561-756, Korea \\ ${ }^{2}$ Department of Physics, Chonbuk National University, Jeon-ju 561-756, Korea \\ ${ }^{3}$ Institute of Photonics and Information Technology, Chonbuk National University, Jeon-ju 561-756, Korea \\ ${ }^{4}$ Jiny Photonics Co. Ltd., Wan-ju 565-854, Korea
}

(Received April 24, 2012 : revised August 17, 2012 : accepted August 20, 2012)

\begin{abstract}
A polarimetric experimental method was developed to determine the Jones matrix elements of transparent optical materials without sign ambiguity. A set of polarization dependent transmittance data of the samples was measured with polarizer - sample - analyzer system and another set of data was measured with polarizer - sample - quarter-wave plate - analyzer. Two data sets were compared and mathematically analyzed to obtain the correct signs of the elements of the matrix. The Jones matrix elements of a quarter-wave plate were determined to check the validity of the method. The experimentally obtained matrix elements of the quarter-wave plate were consistent with the theoretical expectations. The same method was applied to obtain the Jones matrix elements of a twisted nematic liquid crystal panel.
\end{abstract}

Keywords : Jones matrix element, Liquid crystal, Spatial light modulator, Polarimetry

OCIS codes : : (230.6120) Spatial light modulators; (120.5410) Polarimetry; (160.3710) Liquid crystals

\section{INTRODUCTION}

Liquid crystal panels (LCPs) are optoelectronic devices that can modulate the state of light. They are used for many practical applications including flat panel display, holography and optical information processing because of their inherent ability to spatially modulate a light beam in a programmable manner [1-4]. Twisted nematic (TN) type LCP spatial light modulators (SLMs) are good for intensity modulation and parallel aligned LCP SLMs are suitable for phase modulation. Some application areas of SLMs such as ultra-short laser pulse control require phase modulation $[5,6]$. However parallel aligned LCP SLMs are expensive, while TN-LCP SLMs are relatively low-cost and easily available. This is why much effort has been made to use TN-LCP SLMs for many applications $[7,8]$.

For programmable operation of a TN-LCP spatial light modulator (SLM), the Jones matrix of the TN-LCP needs to be determined accurately. However, manufacturers do not usually provide that information, and the user must determine the matrix. According to the literature, the TN-LCP can be considered as a stack of thin slices acting as homogeneous uniaxial crystals [9]. The Jones matrix of a homogeneous uniaxial crystal slice can be written as a function of its ordinary and extraordinary indices of refraction as well as the orientation angle of the liquid crystal molecules [9]. The Jones matrix of the whole TN-LCP can be described as the product of the individual Jones matrices of each homogeneous uniaxial crystal slice [10]. According to the approximation, the Jones matrix of the whole TN-LCP can be written as

$$
J=c_{1} \cdot e^{-i \Delta \phi}\left(\begin{array}{ll}
f-i g & -h-i j \\
h-i j & f+i g
\end{array}\right)
$$

Here $c_{1}$ is a constant used to define the intensity loss, $i$ is $\sqrt{-1}$, and $\Delta \phi$ is the phase change induced by the LCP at a given wavelength. The matrix describes the change in polarization state of the incoming beam. The goal of this study is to determine the signs as well as the absolute

\footnotetext{
*Corresponding author: mwkim@chonbuk.ac.kr

Color versions of one or more of the figures in this paper are available online.
} 
values of the matrix elements can be determined by polarimetric methods experimentally, but it has been thought that the signs of the elements can not be determined uniquely by polarimetric methods only. However it has been shown that the signs can be determined by polarimetric methods [11].

In this paper, we demonstrated a polarimetric method for unambiguous determination of signs of the Jones matrix elements. By measuring the transmittance with an optical system of polarizer-LCP-analyzer, we obtained the set of the unitary matrix elements. In addition, by measuring the transmittance after inserting a quarter wave plate in the system, we obtained another set of unitary matrix elements. By comparing two sets of unitary matrix elements, we could determine the unitary matrix elements accurately without the sign ambiguity.

Our method has some advantages with respect to other previous similar methods. First, our method improved the accuracy. In particular, polarizer and analyzer rotate in the same and opposite directions with 1degree angular increment from $0^{\circ}$ to $180^{\circ}$, so Jones matrix elements were obtained from fitting a trigonometric function with many data points. Also, our method uses only two rotating elements (polarizer and analyzer) and except for the rotation of the polarizers the alignments of all the other optical elements were not disturbed during the measurement, which can reduce potential errors due to realignment.

\section{THEORY}

\subsection{Transmittance Intensity of the First Part of the} Optical System (Quarter Wave Plate + Polarizer)

The polarimetric method to determine the Jones matrix is based on the transmittance measurement of the optical system. Let us first explain how to calculate the transmittance of the optical system. Our whole optical system consists of a He-Ne laser light source, a quarter wave plate (QWP1), polarizer (P), TN-LCP, analyzer (A) and a silicon photo-diode detector as shown in Fig. 1.

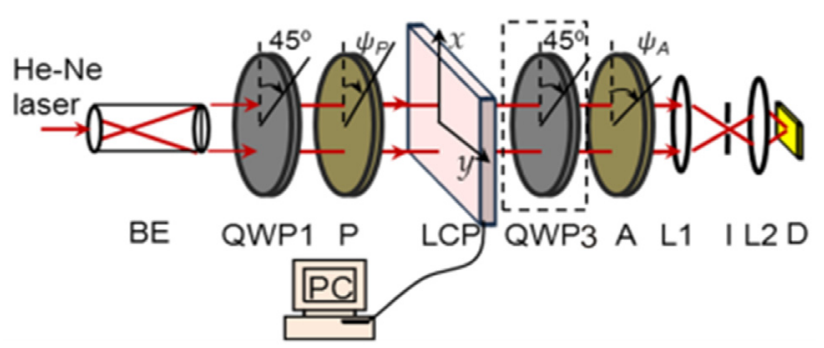

FIG. 1. The optical system used to measure the transmittance of a TN-LCP. Azimuth angles of the polarizer and analyzer are $\psi_{P}$ and $\psi_{A}$, respectively. BE: beam expander, QWP1 and QWP3: quarter wave plates, P: polarizer, LCP: liquid crystal panel, A: analyzer, L: lens, I: Iris, D: photodiode.
First we considered the transmittance intensity of the optical system consists of a light source, a quarter-wave plate (QWP1) and a polarizer. The laser light source was linearly polarized, so the QWP1 was used to make the linearly polarized light into the circularly polarized light. The circularly polarized light was the input beam of the polarizer. The azimuth angle of the polarizer was defined as $\psi_{P}$. The relation between the Jones vector of the input beam $\left(a, b \cdot e^{i \delta}\right)$ and that of the output beam $\left(x_{\text {out } 1}, y_{\text {out } 1}\right)$ for the optical system can be written as Eq. (2)

$$
\begin{aligned}
& \left(\begin{array}{l}
x_{\text {out } 1} \\
y_{\text {out } 1}
\end{array}\right)=P\left(\psi_{P}\right) \cdot Q\left(\frac{\pi}{4}\right) \cdot\left(\begin{array}{c}
a \\
b \cdot e^{i \delta}
\end{array}\right) \\
& =e^{-i \psi_{P}} \cdot\left(a-i b \cdot e^{-i\left(\delta+2 \psi_{P}\right)}\right) \cdot\left(\begin{array}{l}
\cos \left(\psi_{A}\right) \\
\sin \left(\psi_{A}\right)
\end{array}\right),
\end{aligned}
$$

Here $a$ and $b$ are the electric field components on the vertical $(x, z)$ and horizontal $(y, z)$ planes, respectively. $\delta$ is a phase difference between the electric field components. $Q(\pi / 4)$ is the Jones matrix of the QWP1 whose fast axis was aligned $45^{\circ}$ with respect to the vertical $(x)$ axis. $P\left(\psi_{P}\right)$ is the Jones matrix of the polarizer whose azimuthal angle is $\psi_{P}$. The output beam intensity was determined by following equation,

$$
I_{1}=\left(\begin{array}{ll}
x_{\text {out } 1}^{*} & y_{\text {out } 1}^{*}
\end{array}\right) \cdot\left(\begin{array}{l}
x_{\text {out } 1} \\
y_{\text {out } 1}
\end{array}\right)=a^{2}+b^{2}+2 \cdot a \cdot b \cdot \sin \left(\delta+2 \psi_{P}\right) .
$$

The laser light was almost linearly polarized in the vertical $(x, z)$ plane, so the electric field components satisfied a condition, $a \gg b$.

\subsection{Transmittance Intensity of the Whole Optical System (Quarter Wave Plate + Polarizer + TN-LCP + Analyzer)}

We calculated the transmittance intensity of the whole optical system consists of a light source, a quarter-wave plate (QWP1), a polarizer, a TN-LCP and an analyzer. The TN-LCP was placed between a polarizer and an analyzer whose azimuth angles were $\psi_{P}$ and $\psi_{A}$, respectively. The output beam of the polarizer described in Eq. (2) was used as the input beam of the later optical system. The Jones vector of the output beam for the whole optical system can be written as Eq. (4).

$$
\begin{aligned}
\left(\begin{array}{l}
x_{\text {out }} \\
y_{\text {out }}
\end{array}\right) & =A\left(\psi_{A}\right) \cdot J \cdot P\left(\psi_{P}\right) \cdot Q\left(\frac{\pi}{4}\right) \cdot\left(\begin{array}{c}
a \\
b \cdot e^{i \delta}
\end{array}\right) \\
& =c \cdot e^{-i\left(\Delta \phi+\psi_{P}\right)} \cdot\left(a-i b \cdot e^{-i\left(\delta+2 \psi_{P}\right)}\right) \cdot \widetilde{T}_{\psi_{P}, \psi_{A}} \cdot\left(\begin{array}{l}
\cos \left(\psi_{A}\right) \\
\sin \left(\psi_{A}\right)
\end{array}\right),
\end{aligned}
$$

$$
I=c_{2} \cdot\left\{a^{2}+b^{2}+2 \cdot a \cdot b \cdot \sin \left(\delta+2 \psi_{P}\right)\right\} \cdot\left|\tilde{T}_{\psi_{P}, \psi_{A}}\right|
$$




$$
\begin{aligned}
\tilde{T}_{\psi_{P}, \psi_{A}}=f \cos \left(\psi_{A}-\psi_{P}\right)+h \sin \left(\psi_{A}-\psi_{P}\right) \\
-i\left\{g \cos \left(\psi_{A}+\psi_{P}\right)+j \sin \left(\psi_{A}+\psi_{P}\right)\right\},
\end{aligned}
$$

\subsection{Definition of the Transmittance of the Optical System Composed of TN-LCP and Analyzer}

We defined the transmittance of the optical system composed of TN-LCP and analyzer, $T_{\psi_{P}, \psi_{A}}$, as the relative ratio between the output (I) beam intensity of Eq. (5) and the input beam intensity $\left(\mathrm{I}_{1}\right)$ of Eq. (3). $T_{\psi_{p} \psi_{A}}$ can be expressed as Eq. (7),

$$
T_{\psi_{P}, \psi_{A}}=\frac{I}{I_{1}}=c_{3} \cdot\left|\tilde{T}_{\psi_{P}, \psi_{A}}\right|^{2}
$$

Here $c_{3}$ is a constant.

\section{EXPERIMENT}

The optical system used in our study was shown in Fig. 1. The light source was a linearly polarized He-Ne laser. The laser beam was expanded by using the beam expander (BE) and then was sent to the quarter wave plate (QWP1). The quarter-wave plate made the linearly polarized light circularly polarized. The circularly polarized beam was directed to a rotating linear polarizer $(\mathrm{P})$ and then was sent to the sample. The polarization state of the transmitted beam was investigated with an analyzer (A) and an additional quarter wave plate (QWP3). The intensity of the beam passed through the analyzer was measured with the photodiode (D). A quarter-wave plate (QWP2) and a TN-LCP SLM (Holoeye LC2002, SONY LCX016AL-6) were used as samples.

The transmittance of the optical system (sample + analyzer) depends only on the sum $\left(\psi_{A}+\psi_{P}\right)$ and the difference $\left(\psi_{A}\right.$ $-\psi_{P}$ ) of the angles of the polarizer and analyzer. After substituting $\varphi=\psi_{A}-\psi_{P}$ and $\psi=\psi_{A}+\psi_{P}$ into Eq. (7), the transmittance of the optical system, $T_{\varphi, \psi}$ takes a simpler form.

$$
T_{\varphi, \psi}=C+A \cos \left(2 \varphi+2 \varphi_{0}\right)+B \cos \left(2 \psi+2 \psi_{0}\right)
$$

Here $\mathrm{C}$ is a constant. $\varphi_{0}, \psi_{0}$ are the initial phases of the trigonometric functions and $\mathrm{A}, \mathrm{B}$ are the amplitudes of the functions. A set of transmittance $T_{\varphi, \psi}$ data was collected by changing $\varphi$ and $\psi$ from $0^{\circ}$ to $360^{\circ}$. The coefficients $\mathrm{A}$ and $\mathrm{B}$, also the initial phase angles $\varphi_{0}$ and $\psi_{0}$ in Eq. (8) were determined by the Newton-Raphson fitting method.

\section{RESULTS AND DISCUSSION}

\subsection{Determination of the Jones Matrix Elements of a Quarter-wave Plate}

To check the validity of our method, the Jones matrix elements of a quarter wave plate (QWP2) is experimentally determined and compared with the theoretical one. The fast axis of the QWP2 is set to make $45^{\circ}$ with the $x$ axis. If the quarter-wave plate is ideal, then the Jones matrix should be

$$
J_{Q W P}=c_{4}\left(\begin{array}{cc}
f-i g & -h-i j \\
h-i j & f+i g
\end{array}\right)=c_{4}\left(\begin{array}{cc}
1 / \sqrt{2} & -i / \sqrt{2} \\
-i / \sqrt{2} & 1 / \sqrt{2}
\end{array}\right)
$$

from Eq. (1), where $c_{4}$ is the constant representing the intensity loss. The matrix should be unitary, so the elements $f, h, g$ and $j$ should satisfy the condition, $f^{2}+h^{2}$ $+g^{2}+j^{2}=1$. To satisfy the constraints $f, j, h$ and $g$ should be $f=j=1 / \sqrt{2}=0.707, h=g=0$. Eqs. (6), (7), and (8) show that $f, j, h$ and $g$ are connected to the coefficients $\mathrm{A}$ and $\mathrm{B}$ also the initial phase angles $\varphi_{0}$ and $\psi_{0}$ of the trigonometric functions representing the transmittance of the optical system, i.e.

$$
\begin{aligned}
& f=\sqrt{A} \cos \left(\varphi_{0}\right), h=-\sqrt{A} \sin \left(\varphi_{0}\right), \\
& g=\sqrt{B} \cos \left(\psi_{0}\right) \text { and } j=-\sqrt{B} \sin \left(\psi_{0}\right),
\end{aligned}
$$

The transmittance $T_{\varphi, \psi}$ of the quarter-wave plate (QWP2) and the analyzer was measured and fitted with Eq. (8) to determine $\mathrm{A}, \mathrm{B}, \varphi_{0}$ and $\psi_{0}$. To make Eq. (8) simpler, some conditions are chosen to make the cosines of the sum and the difference of the angles constant. First the analyzer and polarizer rotated in the opposite direction with same angle increment, so that the 'sum angle $\left(\psi_{A}+\psi_{P}\right)$ ' is set to be zero, i.e. $\psi=\psi_{A}+\psi_{P}=0$. Then the transmittance as a function of the difference angle $\varphi=\psi_{A}-\psi_{P}$ is measured as presented in Fig. 2(a). By fitting the data points with a
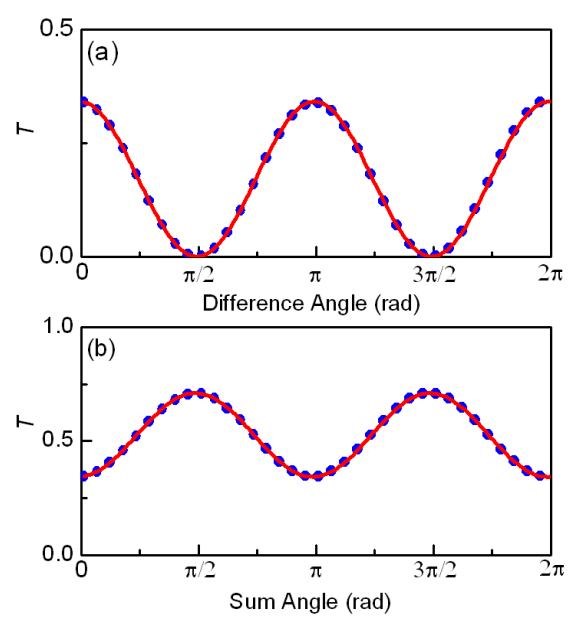

FIG. 2. Transmittance ( $T$ ) of the optical system composed of a quarter-wave plate (QWP2), and an analyzer. Instead of the LCP shown in FIG. 1, a quarter wave plate (QWP2) was inserted. (a) The analyzer and the polarizer rotated in the opposite directions. (b) The analyzer and polarizer rotated together. The solid circles are the experimental result, and the line is the theoretical estimation. 
cosine function, the information about $f$ and $h$ is obtained.

Similarly, the analyzer and the polarizer are rotated in the same direction with the same angle increment so that the 'difference angle $\left(\psi_{A^{-}}-\psi_{P}\right)$ ' is set to be zero, i.e. $\varphi=\psi_{A}$ $-\psi_{P}=0$. Then the transmittance as a function of the sum angle $\psi=\psi_{A}+\psi_{P}$ is measured as presented in Fig. 2(b). By fitting the data points with a cosine function, the information about $g$ and $j$ is obtained.

It should be noted however that, the relative signs of $g$ and $j$ elements to those of $f$ or $h$ were not uniquely defined because of the ambiguity of the phase of the trigonometric functions $\varphi_{0}$ and $\psi_{0}$ in Eq. (10).

To eliminate the sign ambiguity, an additional quarter-wave plate (QWP3) was inserted between the quarter-wave plate (QWP2) and the analyzer. The fast axis of QWP3 was aligned $45^{\circ}$ with respect to the $x$ axis. Including the contribution of the QWP3, the transmittance became a function of $\varphi$ and $\psi$ as shown in Eq. (11).

$$
\begin{aligned}
T_{\varphi, \psi}= & c_{5} \mid m \cos \left(\psi_{A}-\psi_{P}\right)+q \sin \left(\psi_{A}-\psi_{P}\right)-i\left\{p \cos \left(\psi_{A}+\psi_{P}\right)\right. \\
& \left.l \sin \left(\psi_{A}+\psi_{P}\right)\right\}\left.\right|^{2}=c_{5}\left\{(m \cos (\varphi)+q \sin (\varphi))^{2}+(p \cos (\psi)+l \sin (\psi))^{2}\right\},
\end{aligned}
$$

Here $c_{5}$ is a constant. $m, q, p$ and $l$ were connected to $f$, $h, g$ and $j$ by comparing Eq. (11) with Eq. (6). As a result,

$$
m=f-j, q=h-g, p=h+g, l=f+j
$$

Also the sum of the squares of the new elements should
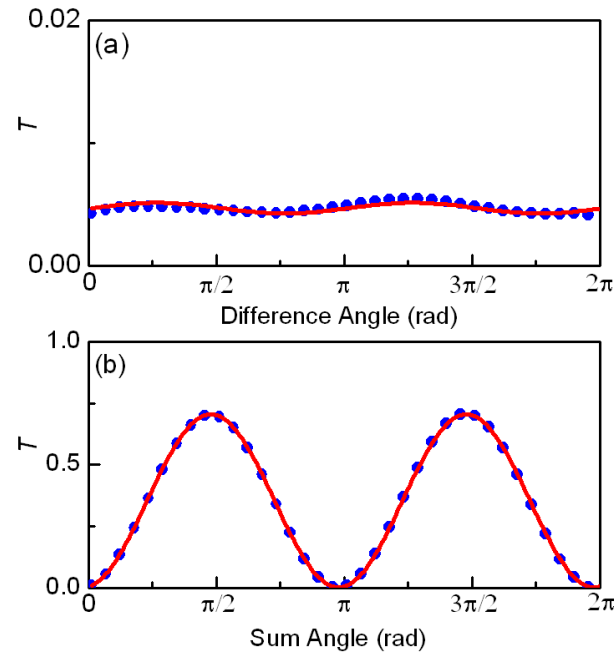

FIG. 3. Transmittance $(T)$ of the optical system composed of a quarter-wave plate (QWP2), additional quarter wave plate (QWP3), and an analyzer. Instead of LCP shown in FIG. 1, a quarter wave plate (QWP2) was inserted. (a) The analyzer and the polarizer rotated in the opposite directions. (b) The analyzer and the polarizer rotated together. The solid circles are the experimental result, and the line is theoretical estimation. satisfy $m^{2}+q^{2}+p^{2}+l^{2}=2$ because of the unitarity.

To determine $m, p, q$ and $l$, first the analyzer and the polarizer are rotated in the opposite directions with the same angle increment so that the sum angle remains zero, i.e. $\psi=\psi_{A}+\psi_{P}=0$. The transmittance as a function of the difference angle $\varphi=\psi_{A}-\psi_{P}$ is presented in Fig. 3(a). By data fitting, the information about $m$ and $q$ is obtained. Similarly, the analyzer and the polarizer are rotated together so that the difference angle remains zero, i.e. $\varphi=\psi_{A}-\psi_{P}=0$. Then the transmittance as a function of the sum angle $\psi=\psi_{A}+\psi_{P}$ is presented in Fig. 3(b). By data fitting, the information about $p$ and $l$ is obtained.

The sign of the $f, g, j$, and $h$ can be determined by comparing four element values of the first method (without QWP3) with four element values of the second method (with QWP3). The experimentally obtained Jones matrix elements of QWP2 are $f=0.706, j=0.707, h=-0.017$ and $g=0.026$ consistent with the theory, which confirms the validity of our method.

\subsection{Determination of the Jones Matrix Elements of a TN-LCP Spatial Light Modulator}

The same method was used to determine the Jones matrix elements of a programmable TN-LCP SLM. A programmable TN-LCP SLM was installed between the polarizer and analyzer as shown in Fig. 1. The TN-LCP can change the intensity and the phase of the input beam as the grey level is controlled by an external device. The corrected transmittances $T_{0}$ of the TN-LCP and the analyzer for several different grey levels were measured. The meaning of the corrected transmittance $T_{0}$ will be discussed in subsection 4.3. Fig. 4 shows the difference angle $\left(\varphi=\psi_{A}-\psi_{P}\right)$ and the sum angle $\left(\psi=\psi_{A}+\psi_{P}\right)$ dependence of the $T_{0}$ for
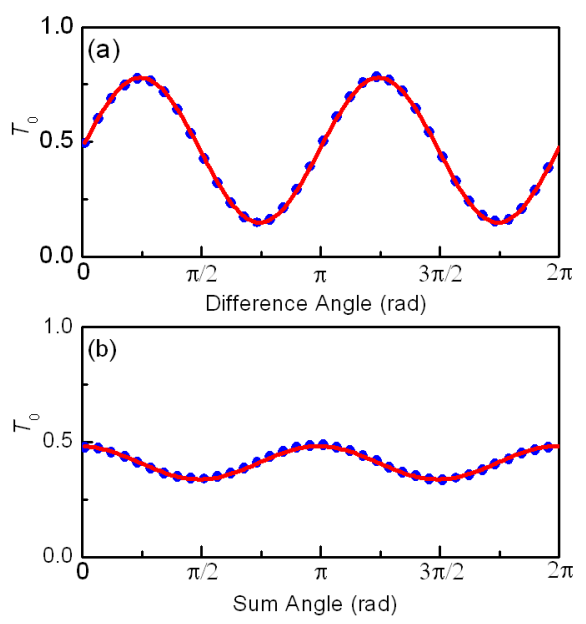

FIG. 4. The corrected transmittance $\left(T_{0}\right)$ of the optical system composed of a TN-LCP, and an analyzer as shown in FIG. 1. (a) The analyzer and polarizer rotated in the opposite directions. (b) The analyzer and the polarizer rotated together. The solid circles are the corrected experimental result, and the line is the theoretical estimation. 

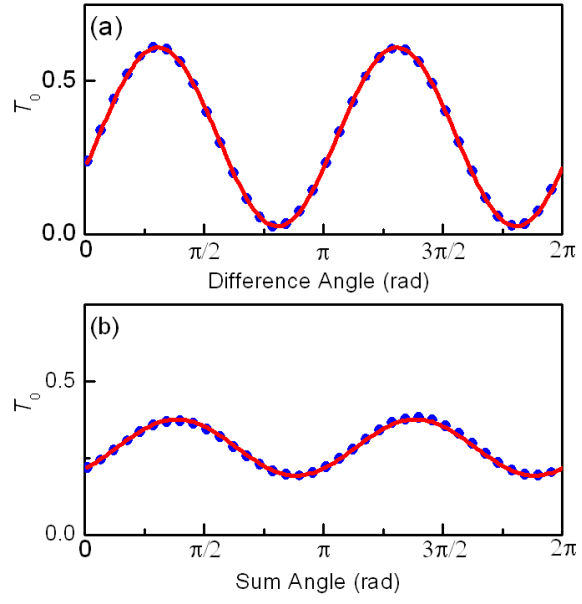

FIG. 5. The corrected transmittance $\left(T_{0}\right)$ of the optical system composed of a TN-LCP, a quarter-wave plate (QWP3), and an analyzer as shown in FIG. 1. (a) The analyzer and the polarizer rotated in the opposite directions. (b) The analyzer and the polarizer rotated together. The solid circles are the corrected experimental result, and the line is the theoretical estimation.

one of the grey levels (grey level 192 of our LCP device). As demonstrated for the quarter-wave plate (QWP2) case, the absolute values of the $f, g, j$, and $h$ of the Jones matrix were determined by fitting the transmittance with Eq. (8).

To determine the signs of the Jones matrix elements, additional set of data were measured. Fig. 5 shows the sum angle $\left(\psi=\psi_{A}+\psi_{P}\right)$ and the difference angle $\left(\varphi=\psi_{A}-\psi_{P}\right)$ dependence of $T_{0}$. Note that additional quarter-wave plate QWP3 was inserted between the TN-LCP and the analyzer for obtaining the data set. The absolute values of the $m, q$, $p$, and $l$ of the Jones matrix were determined by fitting the transmittance with the Eq. (11).

The sign of the $f, g, j$, and $h$ can be determined by comparing four element values of the first method (without QWP3) with four element values of the second method (with QWP3). The experimentally obtained Jones matrix elements of TN-LCP (at grey level 192 of our LCP device) were $f=0.64, h=0.63, g=-0.44$ and $j=-0.02$. The matrix elements of other grey levels can be obtained similarly.

\subsection{Corrections for the Transmittance Measurement}

There is discrepancy between the theoretical transmittances described by Eqs. (8) and (11) and the measured transmittance. Such discrepancy is negligible for the quarter-wave (QWP2), but is not negligible for the TN-LCP. Fig. 6 shows the measured transmittance ( $T$, solid asterisks) of TN-LCP, the error-corrected transmittance ( $T_{0}$, solid circles), and the theoretical fitting function (solid line). They do not overlap perfectly and the discrepancy is especially large around the $\pi / 4$ of the difference angle.

The intensity loss occurred at the TN-LCP is the most

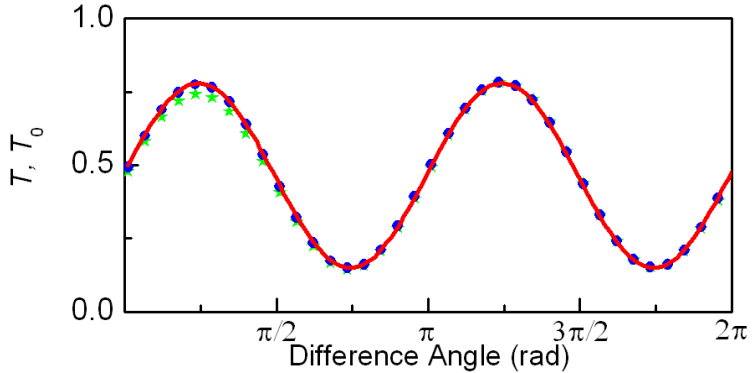

FIG. 6. Uncorrected and corrected transmittance of the optical system composed of a TN-LCP, and an analyzer as shown in FIG. 1. The solid asterisks are the transmittance without correction $(T)$. The analyzer and polarizer rotated in the opposite directions. The solid circles are the transmittance $\left(T_{0}\right)$ of the optical system with correction, and the line is the theoretical estimation.
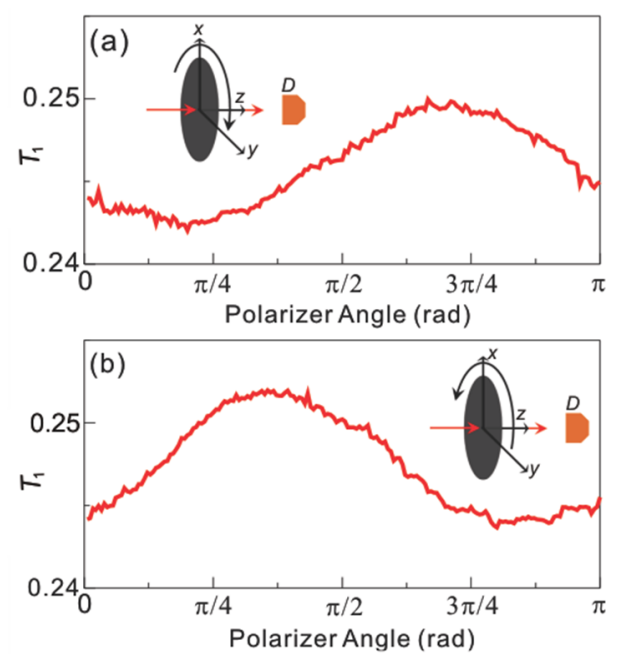

FIG. 7. Transmittance $\left(T_{1}\right)$ of the TN-LCP for (a) the clockwise polarizer rotation and (b) for the counterclockwise polarizer rotation as illustrated in the insets. ' $D$ ' represents the detector.

dominant reason of the discrepancy. The intensity loss can be caused by many factors including absorption, scattering or reflection. However, the intensity loss does not affect the fitting process significantly in our method.

Figure 7 shows the polarizer angle dependence of the transmittance of the TN-LCP $\left(T_{1}\right)$. Note that $T_{1}$ was measured without the analyzer and the additional quarter-wave plate QWP3. There are two polarizer rotation directions. Fig. 7(a) shows the $\mathrm{T} 1$ for the clockwise polarizer rotation (See the inset of Fig. 7(a)). This corresponds to the polarize rotation for the difference angle $\left(\varphi=\psi_{A}-\psi_{P}\right)$ measurement case shown in Figs. 4(a) and 5(a). Fig. 7(b) shows the $T_{1}$ for the counter-clockwise polarizer rotation (See the inset of Fig. 7(b)). This corresponds to the polarize rotation for the sum angle $\left(\psi=\psi_{A}+\psi_{P}\right)$ measurement case shown in Figs. 4(b) and 5(b). The $T_{1}$ data show the oscillating 
behavior deviated from the constant value about $2 \%$.

The final transmittance $(T)$ of the whole optical system can be considered as the multiplication of the $T_{1}$ and the transmittance $\left(T_{0}\right)$ of the analyzer (or analyzer + the additional quarter-wave plate QWP3, when QWP3 is inserted). To remove the polarizer dependence in the final transmittance of the whole optical system the transmittance $T$ was divided with transmittance $\left(T_{1}\right)$ of TN-LCP. This is the corrected transmittance $\left(T_{0}=T / T_{1}\right)$ of the optical system also the transmittance $\left(T_{0}\right)$ of the analyzer (or analyzer + the additional quarter-wave plate QWP3). Errors were negligible for the quarter-wave plate QWP2 data, so the Jones matrix elements of QWP2 are obtained without the correction.

\section{CONCLUSION}

A polarimetric method was developed for determining the Jones matrix element without the sign ambiguity. The credibility of the method was checked by comparing the theoretical and experimental Jones matrix elements of a quarter-wave plate. The method was used to determine the Jones matrix elements of a twisted nematic liquid crystal panel (TN-LCP) successfully. The proposed method can be used to determine the Jones matrix elements of any other transparent optical materials.

\section{ACKNOWLEDGMENT}

This research was supported by the National Research Foundation of Korea (Grants No. 2010-0023535 and No 2202011-1-C00016).

\section{REFERENCES}

1. J. A. Castellano, "Modifying light," American Scientist 94, 438-445 (2006).

2. T. H. Barnes, K. Matsuda, T. Eiju, K. Matsumoto, and F. Johnson, "Joint transform correlator using a phase only spatial light modulator,” Jpn. J. Appl. Phys. 29, 1293-1296 (1990).

3. F. Mok, J. Diep, H. Liu, and D. Psaltis, "Real-time computergenerated hologram by means of liquid-crystal television spatial light modulator," Opt. Lett. 11, 748-750 (1986).

4. A. S. Ostrovsky, G. Martínez-Niconoff, V. Arrizón, P. MartínezVara, M. A. Olvera-Santamaría, and C. Rickenstorff-Parrao, "Modulation of coherence and polarization using liquid crystal spatial light modulators," Opt. Express 17, 5257-5264 (2009).

5. Z. Wang, D. L. Marks, P. S. Carney, L. J. Millet, M. U. Gillette, A. Mihi, P. V. Braun, Z. Shen, S. G. Prasanth, and G. Popescu, "Spatial light interference tomography," Opt. Express 19, 19907-19918 (2011).

6. L. Polachek, D. Oron, and Y. Silberberg, "Full control of the spectral polarization of ultrashort pulses," Opt. Lett. 31, 631-633 (2006).

7. D. A. Yocky, T. H. Bernes, K. Matsumoto, N. Ooyama, and K. Matsuda, "Simple measurement of the phase modulation capability of liquid crystal phase-only light modulators," Optik 84, 140-144 (1990).

8. K. Ohkubo and J. Ohtsubo, "Evaluation of LCTV as a spatial light modulator," Opt. Commun. 102, 116-124 (1993).

9. K. Lu and B. E. A. Saleh, "Theory and design of the liquid crystal TV as an optical phase modulator," Opt. Eng. 29, 240-246 (1990).

10. A. Yariv and P. Yeh, Optical Waves in Crystal (Wiley, New York, USA, 1984), Chapter 5.

11. I. Moreno, P. Velásquez, C. R. Fernández-Pousa, M. M. Sánchez-López, and F. Mateos, "Jones matrix method for predicting and optimizing the optical modulation properties of a liquid-crystal display,” J. Appl. Phys. 94, 3697 (2003). 